\title{
Inverse problem for a time-fractional parabolic equation
}

\author{
Ebru Ozbilge ${ }^{1 *}$ and Ali Demir ${ }^{2}$
}

\author{
"Correspondence: \\ ebru.ozbilge@ieu.edu.tr \\ ${ }^{1}$ Department of Mathematics, \\ Faculty of Science and Literature, \\ Izmir University of Economics, \\ Sakarya Caddesi, No. 156, Balcova, \\ Izmir, 35330, Turkey \\ Full list of author information is \\ available at the end of the article
}

\begin{abstract}
This article deals with the mathematical analysis of the inverse coefficient problem of identifying the unknown coefficient $k(x)$ in the linear time-fractional parabolic equation $D_{t}^{\alpha} u(x, t)=\left(k(x) u_{x}\right)_{x}+q u_{x}(x, t)+p(t) u(x, t), 0<\alpha \leq 1$, with mixed boundary conditions $k(0) u_{x}(0, t)=\psi_{0}(t), u(1, t)=\psi_{1}(t)$. By defining the input-output mappings $\Phi[\cdot]: \mathcal{K} \rightarrow C[0, T]$ and $\Psi[\cdot]: \mathcal{K} \rightarrow C^{1}[0, T]$ the inverse problem is reduced to the problem of their invertibility. Hence the main purpose of this study is to investigate the distinguishability of the input-output mappings $\Phi[\cdot]$ and $\Psi[\cdot]$. This work shows that the input-output mappings $\Phi[\cdot]$ and $\Psi[\cdot]$ have distinguishability property. Moreover, the value $k(1)$ of the unknown diffusion coefficient $k(x)$ at $x=1$ can be determined explicitly by making use of measured output data (boundary observation) $k(1) u_{x}(1, t)=h(t)$, which brings about a greater restriction on the set of admissible coefficients. It is also shown that the measured output data $f(t)$ and $h(t)$ can be determined analytically by a series representation. Hence the input-output mappings $\Phi[\cdot]: \mathcal{K} \rightarrow C[0, T]$ and $\Psi[\cdot]: \mathcal{K} \rightarrow C^{1}[0, T]$ can be described explicitly, where $\Phi[k]=\left.u(x, t ; k)\right|_{x=0}$ and $\Psi[k]=\left.k(x) u_{x}(x, t ; k)\right|_{x=1}$.
\end{abstract}

\section{Introduction}

The inverse problem of determining unknown coefficient in a linear parabolic equation by using over-measured data has generated increasing interest from engineers and scientists during the last few decades. This kind of problem plays a crucial role in engineering, physics, and applied mathematics. The problem of recovering unknown coefficient(s) in the mathematical model of a physical phenomenon is frequently encountered. Intensive study has been carried out on this kind of problem, and various numerical methods were developed in order to overcome the problem of determining unknown coefficients [1-27]. The inverse problem of an unknown coefficient in a quasi-linear parabolic equation has been studied by Demir and Ozbilge [1,2]. Moreover, the existence and uniqueness of solutions for fractional differential equations with nonlocal and integral boundary conditions have been studied by Ashyralyev and Sharifov. Also, finite difference methods for fractional parabolic and hyperbolic differential equations with various conditions have been studied by Ashyralyev et al. [3-8]. Second order implicit finite difference schemes have been applied to the right-hand side of the identification problem by Erdogan and Ashyralyev [9].

Fractional differential equations are generalizations of ordinary and partial differential equations to an arbitrary fractional order. By linear time-fractional parabolic equation,

C) 2015 Ozbilge and Demir; licensee Springer. This is an Open Access article distributed under the terms of the Creative Commons Attribution License (http://creativecommons.org/licenses/by/4.0), which permits unrestricted use, distribution, and reproduction in any medium, provided the original work is properly credited. 
we mean certain parabolic-like partial differential equation governed by master equations containing fractional derivatives in time $[10,11]$. The research areas of fractional differential equations range from theoretical to applied aspects. The main goal of this study is to investigate the inverse problem of determining unknown coefficient $k(x)$ in a onedimensional time fractional parabolic equation. We first obtain the unique solution of this problem using Fourier method of separation of variables with respect to the eigenfunctions of a corresponding Sturm-Liouville eigenvalue problem under certain conditions [12]. As the next step, the noisy free measured output data are used to introduce the input-output mappings $\Phi[\cdot]: \mathcal{K} \rightarrow C[0, T]$ and $\Psi[\cdot]: \mathcal{K} \rightarrow C^{1}[0, T]$. Finally we investigate the distinguishability of the unknown coefficient via the above input-output mappings $\Phi[\cdot]$ and $\Psi[\cdot]$.

Consider now the following initial boundary value problem:

$$
\left\{\begin{array}{l}
D_{t}^{\alpha} u(x, t)=\left(k(x) u_{x}(x, t)\right)_{x}+q u_{x}(x, t)+p(t) u(x, t), \quad 0<\alpha \leq 1,(x, t) \in \Omega_{T} \\
u(x, 0)=g(x), \quad 0<x<1, \\
k(0) u_{x}(0, t)=\psi_{0}(t), \quad u(1, t)=\psi_{1}(t), \quad 0<t<T
\end{array}\right.
$$

where $\Omega_{T}=\left\{(x, t) \in \mathbb{R}^{2}: 0<x<1,0<t \leq T\right\}$ and the fractional derivative $D_{t}^{\alpha} u(x, t)$ is defined in the Caputo-Dzherbashyan sense, that is, $D_{t}^{\alpha} u(x, t)=\left(I^{1-\alpha} u^{\prime}\right)(t), 0<\alpha \leq 1, I^{\alpha}$ being the Riemann-Liouville fractional integral,

$$
\left(I^{\alpha} f\right)(t)= \begin{cases}\frac{1}{\Gamma(\alpha)} \int_{0}^{t}(t-\tau)^{\alpha-1} f(\tau) d \tau, & 0<\alpha \leq 1 \\ f(t), & \alpha=0 .\end{cases}
$$

The left and right boundary value functions $\psi_{0}(t)$ and $\psi_{1}(t)$ belong to $C[0, T]$. The functions $0<c_{0} \leq k(x)<c_{1}$ and $g(x)$ satisfy the following conditions:

(C1) $k(x) \in C^{1}[0,1]$.

(C2) $g(x) \in C^{2}[0,1], g^{\prime}(0)=\frac{\psi_{0}(0)}{k(0)}, g(1)=\psi_{1}(0)$.

(C3) $p(t) \in C[0,1]$.

Under these conditions, initial boundary value problem (1) has the unique solution $u(x, t)$ defined in the domain $\bar{\Omega}_{T}=\left\{(x, t) \in \mathbb{R}^{2}: 0 \leq x \leq 1,0 \leq t \leq T\right\}$ which belongs to the space $C\left(\bar{\Omega}_{T}\right) \cap W_{t}^{1}(0, T] \cap C_{x}^{2}(0,1)$. Moreover, it satisfies the equation, initial and boundary conditions. Note that the space $W_{t}^{1}(0, T]$ contains the functions $f \in C^{1}(0, T]$ such that $f^{\prime}(x) \in L(0, T)$.

Consider the inverse problem of determining the unknown coefficient $k(x)$ from the Dirichlet type measured output data at the boundary $x=0$,

$$
u(0, t)=f(t), \quad t \in(0, T]
$$

and the Neumann type measured output data at the boundary $x=1$,

$$
k(1) u_{x}(1, t)=h(t), \quad t \in(0, T] .
$$

Here $u=u(x, t)$ is the solution of parabolic problem (1). The functions $f(t)$ and $h(t)$ are assumed to be noisy free measured output data. In this context, parabolic problem (1) will be referred to as a direct (forward) problem, with the inputs $g(x), k(x), q$, and $p(t)$. It is 
assumed that the functions $f(t), g(t)$ belong to $C[0, T], C^{1}[0, T]$, respectively, and satisfy the consistency conditions $f(0)=g(0)$ and $k(1) g^{\prime}(1)=h(0)$.

Let us denote $\mathcal{K}:=\left\{k(x) \in C^{1}[0,1]: c_{1}>k(x) \geq c_{0}>0, x \in[0,1]\right\} \subset C[0,1]$, the set of admissible coefficients $k(x)$. Also, let us introduce the input-output mappings $\Phi[\cdot]: \mathcal{K} \rightarrow$ $C[0, T]$ and $\Psi[\cdot]: \mathcal{K} \rightarrow C^{1}[0, T]$ where

$$
\Phi[k]=\left.u(x, t ; k)\right|_{x=0}, \quad k \in \mathcal{K}_{0} .
$$

and

$$
\Psi[k]=\left.k(x) u_{x}(x, t ; k)\right|_{x=1}, \quad k \in \mathcal{K}_{1} .
$$

Then the inverse problem with the measured output data $f(t)$ and $h(t)$ can be formulated as follows:

$$
\begin{aligned}
& \Phi[k]=f, \quad f \in C(0, T], \\
& \Psi[k]=h, \quad h \in C^{1}(0, T] .
\end{aligned}
$$

Hence, the inverse problem of determining unknown coefficient $k(x)$ is reduced to the problem of invertibility of the input-output mappings $\Phi[\cdot]$ and $\Psi[\cdot]$. This leads us to investigate the distinguishability of the unknown coefficient via the above input-output mappings.

We say that the mappings $\Phi[\cdot]: \mathcal{K} \rightarrow C[0, T]$ and $\Psi[\cdot]: \mathcal{K} \rightarrow C^{1}[0, T]$ have the distinguishability property if $\Phi\left[k_{1}\right] \neq \Phi\left[k_{2}\right]$ implies $k_{1}(x) \neq k_{2}(x)$ and the same holds for $\Psi[\cdot]$. This, in particular, means injectivity of the inverse mappings $\Phi^{-1}$ and $\Psi^{-1}$. In this paper, measured output data of Neumann type at the boundary $x=1$ are used in the identification of the unknown coefficient. In addition, in the determination of the unknown parameter, analytical results are obtained.

The paper is organized as follows: In Section 2, an analysis of the inverse problem with the single measured output data $f(t)$ at the boundary $x=0$ is given. An analysis of the inverse problem with the single measured output data $h(t)$ at the boundary $x=1$ is considered in Section 3. Finally, some concluding remarks are given in the last section.

\section{An analysis of the inverse problem with given measured data $f(t)$}

Consider now the inverse problem with one measured output data $f(t)$ at $x=0$. In order to formulate the solution of parabolic problem (1) by using the Fourier method of the separation of variables, let us first introduce an auxiliary function $v(x, t)$ as follows:

$$
v(x, t)=u(x, t)-\psi_{1}(t)+(1-x) \frac{\psi_{0}(t)}{k(0)}, \quad x \in[0,1]
$$

by which we transform problem (1) into a problem with homogeneous boundary conditions. Hence initial boundary value problem (1) can be rewritten in terms of $v(x, t)$ in the 
following form:

$$
\left\{\begin{aligned}
& D_{t}^{\alpha} v(x, t)-v_{x x}(x, t)=\left((k(x)-1) v_{x}(x, t)\right)_{x}+k^{\prime}(x) \frac{\psi_{0}(t)}{k(0)}+p(t) v(x, t)+p(t) \psi_{1}(t) \\
&-p(t)(1-x) \frac{\psi_{0}(t)}{k(0)}+q v_{x}(x, t)+q \frac{\psi_{0}(t)}{k(0)}-D_{t}^{\alpha} \psi_{1}(t) \\
&+D_{t}^{\alpha} \frac{\psi_{0}(t)}{k(0)}-x D_{t}^{\alpha} \frac{\psi_{0}(t)}{k(0)}, \quad(x, t) \in \Omega_{T}, \\
& v(x, 0)=g(x)-\psi_{1}(0)+(1-x) \frac{\psi_{0}(0)}{k(0)}, \quad 0<x<1, \\
& k(0) v_{x}(0, t)=0, \quad v(1, t)=0, \quad 0<t<T .
\end{aligned}\right.
$$

The unique solution of the initial boundary value problem can be represented in the following form [12]:

$$
\begin{aligned}
v(x, t)= & \sum_{n=1}^{\infty}\left\langle\zeta(\theta), \phi_{n}(\theta)\right| E_{\alpha, 1}\left(-\lambda_{n} t^{\alpha}\right) \phi_{n}(x) \\
& +\sum_{n=1}^{\infty}\left(\int_{0}^{t} s^{\alpha-1} E_{\alpha, \alpha}\left(-\lambda_{n} s^{\alpha}\right)\left\langle\xi(\theta, t-s), \phi_{n}(\theta)\right\rangle d s\right) \phi_{n}(x),
\end{aligned}
$$

where

$$
\begin{aligned}
& \zeta(x)=g(x)-\psi_{1}(0)+(1-x) \frac{\psi_{0}(0)}{k(0)} \\
& \xi(x, t)=\left((k(x)-1) v_{x}(x, t)\right)_{x}+k^{\prime}(x) \frac{\psi_{0}(t)}{k(0)}+p(t) v(x, t)+p(t) \psi_{1}(t)-p(t)(1-x) \frac{\psi_{0}(t)}{k(0)} \\
&+q v_{x}(x, t)+q \frac{\psi_{0}(t)}{k(0)}-D_{t}^{\alpha} \psi_{1}(t)+D_{t}^{\alpha} \frac{\psi_{0}(t)}{k(0)}-x D_{t}^{\alpha} \frac{\psi_{0}(t)}{k(0)}
\end{aligned}
$$

Moreover, $\left\langle\zeta(\theta), \phi_{n}(\theta)\right\rangle=\int_{0}^{1} \phi_{n}(\theta) \zeta(\theta) d \theta, E_{\alpha, \beta}$ being the generalized Mittag-Leffler function defined by

$$
E_{\alpha, \beta}(z)=\sum_{n=0}^{\infty} \frac{z^{n}}{\Gamma(\beta n+\alpha)} .
$$

Assume that $\phi_{n}(x)$ is the solution of the following Sturm-Liouville problem:

$$
\left\{\begin{array}{l}
-\phi_{x x}(x)=\lambda \phi(x), \quad 0<x<1, \\
\phi_{x}(0)=0, \quad \phi(1)=0 .
\end{array}\right.
$$

The Dirichlet type of measured output data at the boundary $x=0$ can be written in terms of $v(x, t)$ in the following form:

$$
v(0, t)+\psi_{1}(t)-\frac{\psi_{0}(t)}{k(0)}=f(t), \quad t \in(0, T] .
$$

In order to arrange the above solution, let us define the following:

$$
\begin{aligned}
& z_{n}(t)=\left\langle\zeta(\theta), \phi_{n}(\theta)\right| E_{\alpha, 1}\left(-\lambda_{n} t^{\alpha}\right), \\
& w_{n}(t)=\int_{0}^{t} s^{\alpha-1} E_{\alpha, \alpha}\left(-\lambda_{n} s^{\alpha}\right)\left\langle\xi(\theta, t-s), \phi_{n}(\theta)\right\rangle d s .
\end{aligned}
$$


The solution in terms of $z_{n}(t)$ and $w_{n}(t)$ can then be rewritten in the following form:

$$
v(x, t)=\sum_{n=1}^{\infty} z_{n}(t) \phi_{n}(x)+\sum_{n=1}^{\infty} w_{n}(t) \phi_{n}(x) .
$$

Substituting $x=0$ yields

$$
v(0, t)=\sum_{n=1}^{\infty} z_{n}(t) \phi_{n}(0)+\sum_{n=1}^{\infty} w_{n}(t) \phi_{n}(0)
$$

Taking into account the over-measured data $v(0, t)+\psi_{1}(t)-\frac{\psi_{0}(t)}{k(0)}=f(t)$,

$$
f(t)=\psi_{1}(t)-\frac{\psi_{0}(t)}{k(0)}+\sum_{n=1}^{\infty} z_{n}(t) \phi_{n}(0)+\sum_{n=1}^{\infty} w_{n}(t) \phi_{n}(0)
$$

is obtained. Therefore, $f(t)$ can be determined analytically by a series representation.

The set of admissible coefficients can be defined as follows:

$$
\mathcal{K}_{0}:=\left\{k(x) \in C^{1}[0,1]: c_{1}>k(x) \geq c_{0}>0, x \in[0,1]\right\}
$$

The right-hand side of identity (4) defines the input-output mapping $\Phi[k]$ on the set of admissible source functions $\mathcal{K}_{0}$ :

$$
\Phi[k](t):=\psi_{1}(t)-\frac{\psi_{0}(t)}{k(0)}+\sum_{n=1}^{\infty} z_{n}(t) \phi_{n}(0)+\sum_{n=1}^{\infty} w_{n}(t) \phi_{n}(0), \quad \forall t \in[0, T] .
$$

The following lemma implies the relation between the parameters $k_{1}(x), k_{2}(x) \in \mathcal{K}_{0}$ at $x=0$ and the corresponding outputs $f_{j}(t):=u\left(0, t ; k_{j}\right), j=1,2$.

Lemma 1 Let $v_{1}(x, t)=v\left(x, t ; k_{1}\right)$ and $v_{2}(x, t)=v\left(x, t ; k_{2}\right)$ be the solutions of direct problem (2), corresponding to the admissible parameters $k_{1}(x), k_{2}(x) \in \mathcal{K}_{0}$. If the condition $k_{1}(0)=k_{2}(0)=k(0)$, then the outputs $f_{j}(t), j=1,2$, satisfy the following integral identity:

$$
\Delta f(t)=\sum_{n=1}^{\infty} \Delta w_{n}(t) \phi_{n}(0)
$$

for each $t \in(0, T]$ where $\Delta f(t)=f_{1}(t)-f_{2}(t), \Delta w_{n}(t)=w_{n}^{1}(t)-w_{n}^{2}(t)$.

Proof By using identity (4), the measured output data $f_{j}(t):=v(0, t)+\psi_{1}(t)-\frac{\psi_{0}(t)}{k(0)}, j=1,2$, can be written as follows:

$$
\begin{aligned}
& f_{1}(t)=\psi_{1}(t)-\frac{\psi_{0}(t)}{k(0)}+\sum_{n=1}^{\infty} z_{n}^{1}(t) \phi_{n}(0)+\sum_{n=1}^{\infty} w_{n}^{1}(t) \phi_{n}(0), \\
& f_{2}(t)=\psi_{1}(t)-\frac{\psi_{0}(t)}{k(0)}+\sum_{n=1}^{\infty} z_{n}^{2}(t) \phi_{n}(0)+\sum_{n=1}^{\infty} w_{n}^{2}(t) \phi_{n}(0),
\end{aligned}
$$


respectively. Note that the definition of $z_{n}(t)$ implies that $z_{n}^{1}(t)=z_{n}^{2}(t)$. Hence, the difference of these formulas implies the desired result.

The lemma and the definitions of $w_{n}(t)$ and $z_{n}(t)$ given above enable us to reach the following conclusion:

Corollary 1 Let the conditions of Lemma 1 hold. If, in addition,

$$
\left\langle\xi^{1}(x, t)-\xi^{2}(x, t), \phi_{n}(x)\right\rangle=0, \quad \forall t \in(0, T], \forall n=0,1, \ldots
$$

holds, then $f_{1}(t)=f_{2}(t), \forall t \in[0, T]$.

Note that $\left\langle\xi^{1}(x, t)-\xi^{2}(x, t), \phi_{n}(x)\right\rangle \neq 0$ for some $n \in N$ implies that $k_{1}(x) \neq k_{2}(x)$. Hence by Lemma 1 we conclude that $k_{1}(x) \neq k_{2}(x)$. Moreover, it leads us to the following important consequence that the input-output mapping $\Phi[k]$ is distinguishable, i.e. $k_{1}(x) \neq k_{2}(x)$ implies $\Phi\left[k_{1}\right] \neq \Phi\left[k_{2}\right]$.

Theorem 1 Let conditions (C1), (C2) and (C3) hold. Assume that $\Phi[\cdot]: \mathcal{K}_{0} \rightarrow C[0, T]$ is the input-output mapping defined by (5) and corresponding to the measured output $f(t):=$ $u(0, t)$. In this case the mapping $\Phi[k]$ has the distinguishability property in the class of admissible parameters $\mathcal{K}_{0}$, i.e.,

$$
\Phi\left[k_{1}\right] \neq \Phi\left[k_{2}\right], \quad \forall k_{1}, k_{2} \in \mathcal{K}_{0}, k_{1}(x) \neq k_{2}(x) .
$$

\section{An analysis of the inverse problem with given measured data $h(t)$}

Consider now the inverse problem with one measured output data $h(t)$ at $x=1$. Taking into account the over-measured data $k(1)\left(v_{x}(x, t)+\frac{\psi_{0}(t)}{k(0)}\right)=h(t)$,

$$
h(t)=k(1)\left(\frac{\psi_{0}(t)}{k(0)}+\sum_{n=1}^{\infty} z_{n}(t) \phi_{n}^{\prime}(1)+\sum_{n=1}^{\infty} w_{n}(t) \phi_{n}^{\prime}(1)\right)
$$

is obtained. Therefore, $h(t)$ can be determined analytically. Substituting $t=0$ into this yields

$$
h(0)=k(1)\left(\frac{\psi_{0}(0)}{k(0)}+\sum_{n=1}^{\infty} z_{n}(0) \phi_{n}^{\prime}(1)\right) .
$$

Hence we obtain the following explicit formula for the value $k(1)$ of the unknown coefficient $k(x)$ :

$$
k(1)=\frac{h(0)}{\frac{\psi_{0}(0)}{k(0)}+\sum_{n=1}^{\infty} z_{n}(0) \phi_{n}^{\prime}(1)} .
$$

Under the determined value $k(1)$ the set of admissible coefficients can be defined as follows:

$$
\mathcal{K}_{1}:=\left\{k(x) \in C^{1}[0,1]: c_{1}>k(x) \geq c_{0}>0, x \in[0,1], k(1)=\frac{h(0)}{\frac{\psi_{0}(0)}{k(0)}+\sum_{n=1}^{\infty} z_{n}(0) \phi_{n}^{\prime}(1)}\right\} .
$$


The right-hand side of identity (6) defines the input-output mapping $\Psi[k]$ on the set of admissible parameters $\mathcal{K}_{1}$ :

$$
\Psi[k](t):=k(1)\left(\frac{\psi_{0}(t)}{k(0)}+\sum_{n=1}^{\infty} z_{n}(t) \phi_{n}^{\prime}(1)+\sum_{n=1}^{\infty} w_{n}(t) \phi_{n}^{\prime}(1)\right), \quad \forall t \in[0, T] .
$$

The following lemma implies the relation between the parameters $k_{1}(x), k_{2}(x) \in \mathcal{K}_{1}$ at $x=1$ and the corresponding outputs $h_{j}(t):=k(1) u_{x}\left(1, t ; k_{j}\right), j=1,2$.

Lemma 2 Let $v_{1}(x, t)=v\left(x, t ; k_{1}\right)$ and $v_{2}(x, t)=v\left(x, t ; k_{2}\right)$ be the solutions of direct problem (2), corresponding to the admissible parameters $k_{1}(x), k_{2}(x) \in \mathcal{K}_{1}$. The outputs $h_{j}(t)$, $j=1,2$, satisfy the following integral identity:

$$
\Delta h(t)=k(1) \sum_{n=1}^{\infty} \Delta w_{n}(t) \phi_{n}^{\prime}(1)
$$

for each $t \in(0, T]$ where $\Delta h(t)=h_{1}(t)-h_{2}(t), \Delta w_{n}(t)=w_{n}^{1}(t)-w_{n}^{2}(t)$.

Proof By using identity (6), the measured output data $h_{j}(t):=k(1)\left(v_{x}(1, t)+\frac{\psi_{0}(t)}{k(0)}\right), j=1,2$, can be written as follows:

$$
\begin{aligned}
& h_{1}(t)=k(1)\left(\frac{\psi_{0}(t)}{k(0)}+\sum_{n=1}^{\infty} z_{n}^{1}(t) \phi_{n}^{\prime}(1)+\sum_{n=1}^{\infty} w_{n}^{1}(t) \phi_{n}^{\prime}(1)\right), \\
& h_{2}(t)=k(1)\left(\frac{\psi_{0}(t)}{k(0)}+\sum_{n=1}^{\infty} z_{n}^{2}(t) \phi_{n}^{\prime}(1)+\sum_{n=1}^{\infty} w_{n}^{2}(t) \phi_{n}^{\prime}(1)\right),
\end{aligned}
$$

respectively. Note that the definition of $z_{n}(t)$ implies that $z_{n}^{1}(t)=z_{n}^{2}(t)$. Hence, the difference of these formulas implies the desired result.

The lemma and the definitions given above enable us to reach the following conclusion:

Corollary 2 Let the conditions of Lemma 2 hold. If, in addition,

$$
\left\langle\xi^{1}(x, t)-\xi^{2}(x, t), \phi_{n}(x)\right\rangle=0, \quad \forall t \in(0, T], \forall n=0,1, \ldots
$$

holds, then $h_{1}(t)=h_{2}(t), \forall t \in[0, T]$.

Note that $\left\langle\xi^{1}(x, t)-\xi^{2}(x, t), \phi_{n}(x)\right\rangle \neq 0$ for some $n \in N$ implies $k_{1}(x) \neq k_{2}(x)$. Hence by Lemma 2 we conclude that $k_{1}(x) \neq k_{2}(x)$. Moreover, it leads us to the important consequence that the input-output mapping $\Psi[k]$ is distinguishable i.e. $k_{l}(x) \neq k_{2}(x)$ implies $\Psi\left[k_{1}\right] \neq \Psi\left[k_{2}\right]$.

Theorem 2 Let conditions (C1), (C2), and (C3) hold. Assume that $\Psi[\cdot]: \mathcal{K}_{1} \rightarrow C^{1}[0, T]$ is the input-output mapping defined by (8) and corresponding to the measured output $h(t):=$ $k(1) u_{x}(1, t)$. In this case the mapping $\Psi[k]$ has the distinguishability property in the class of admissible parameters $\mathcal{K}_{1}$, i.e.,

$$
\Psi\left[k_{1}\right] \neq \Psi\left[k_{2}\right], \quad \forall k_{1}, k_{2} \in \mathcal{K}_{1}, k_{1}(x) \neq k_{2}(x) .
$$




\section{Conclusion}

The aim of this study was to investigate the distinguishability properties of the inputoutput mappings $\Phi[\cdot]: \mathcal{K}_{0} \rightarrow C[0, T]$ and $\Psi[\cdot]: \mathcal{K}_{1} \rightarrow C^{1}[0, T]$, which are determined by the measured output data at $x=0$ and $x=1$, respectively. In this study, we conclude that the distinguishability of the input-output mappings $\Phi[\cdot]$ and $\Psi[\cdot]$ hold which implies the injectivity of the inverse mappings $\Phi^{-1}$ and $\Psi^{-1}$. This provides the insight that compared to the Dirichlet type, the Neumann type of measured output data is more effective for the inverse problems of determining unknown coefficients. Moreover, the measured output data $f(t)$ and $h(t)$ are obtained analytically by a series representation, which leads to the explicit form of the input-output mappings $\Phi[\cdot]$ and $\Psi[\cdot]$. We also show that the value of the unknown coefficient $k(x)$ at $x=1$ is determined by using the Neumann type of measured output data at $x=1$ which brings about more restrictions on the set of admissible coefficients. However, $k(0)$ is not obtained by Dirichlet type of measured output data at $x=0$. This provides the insight that Neumann type of measured output data is more effective than Dirichlet type for the inverse problem of determining an unknown coefficient. This work advances our understanding of the use of the Fourier method of separation of variables and the input-output mapping in the investigation of inverse problems for fractional parabolic equations. The authors plan to consider various fractional inverse problems in future studies, since the method discussed has a wide range of applications.

Competing interests

The authors declare that they have no competing interests.

Authors' contributions

All authors contributed equally and significantly in writing this paper. All authors read and approved the final manuscript.

\section{Author details}

${ }^{1}$ Department of Mathematics, Faculty of Science and Literature, Izmir University of Economics, Sakarya Caddesi, No. 156 Balcova, Izmir, 35330, Turkey. ${ }^{2}$ Department of Mathematics, Kocaeli University, Umuttepe, Izmit, Kocaeli 41380, Turkey.

\section{Acknowledgements}

We would like to thank to the referees for their valuable comments and corrections. The research was supported partly by the Scientific and Technical Research Council (TUBITAK) of Turkey and Izmir University of Economics.

Received: 6 October 2014 Accepted: 18 February 2015 Published online: 04 March 2015

\section{References}

1. Demir, A, Ozbilge, E: Analysis of a semigroup approach in the inverse problem of identifying an unknown coefficient. Math. Methods Appl. Sci. 31, 1635-1645 (2008)

2. Demir, A, Ozbilge, E: Semigroup approach for identification of the unknown coefficient in a quasi-linear parabolic equation. Math. Methods Appl. Sci. 30, 1283-1294 (2007)

3. Amanov, D, Ashyralyev, A: Initial-boundary value problem for fractional partial differential equations of higher order. Abstr. Appl. Anal. 2012, Article ID 9733102 (2012)

4. Ashyralyev, A, Sharifov, Y: Existence and uniqueness of solutions for the system of nonlinear fractional differential equations with nonlocal and integral boundary conditions. Abstr. Appl. Anal. 2012, Article ID 594802 (2012)

5. Ashyralyev, A, Cakir, Z: FDM for fractional parabolic equations with the Neumann condition. Adv. Differ. Equ. 2013, Article ID 120 (2013)

6. Ashyralyev, A, Hicdurmaz, B: On the numerical solution of fractional Schrodinger differential equations with the Dirichlet condition. Int. J. Comput. Math. 89, 1927-1936 (2012)

7. Ashyralyev, A, Dal, F: Finite Difference and Iteration Methods for Fractional Hyperbolic Partial Differential Equations with the Neumann Condition. Discrete Dyn. Nat. Soc. 2012, Article ID 434976 (2012)

8. Ashyralyev, A, Artykov, M, Cakir, Z: A note on fractional parabolic differential and difference equations. In: AIP Conference Proceedings, vol. 1611, pp. 251-254 (2014)

9. Erdogan, AS, Ashyralyev, A: On the second order implicit difference schemes for a right hand side identification problem. Appl. Math. Comput. 226, 212-229 (2014)

10. Renardy, M, Rogers, RC: An Introduction to Partial Differential Equations. Springer, New York (2004)

11. Showalter, RE: Monotone Operators in Banach Spaces and Nonlinear Partial Differential Equations. Am. Math. Soc., Providence (1997)

12. Luchko, Y: Initial boundary value problems for the one dimensional time-fractional diffusion equation. Fract. Calc. Appl. Anal. 15, 141-160 (2012) 
13. Cannon, JR, Lin, Y: An inverse problem of finding a parameter in a semi-linear heat equation. J. Math. Anal. Appl. 145, 470-484 (1990)

14. Cannon, JR, Lin, Y: Determination of a parameter $p(t)$ in some quasi-linear parabolic differential equations. Inverse Probl. 4, 35-44 (1998)

15. Cannon, JR, Lin, Y: Determination of source parameter in parabolic equations. Meccanica 27, 85-94 (1992)

16. Dehghan, M: Identification of a time-dependent coefficient in a partial differential equation subject to an extra measurement. Numer. Methods Partial Differ. Equ. 21, 621-622 (2004)

17. Demir, A, Hasanov, A: Identification of the unknown diffusion coefficient in a linear parabolic equation by the semigroup approach. J. Math. Anal. Appl. 340, 5-15 (2008)

18. Demir, A, Ozbilge, E: Identification of the unknown boundary condition in a linear parabolic equation. J. Inequal. Appl. 2013, Article ID 96 (2013). doi:10.1186/1029-242X-2013-96

19. Erdogan, AS, Sazaklioglu, AU: A note on the numerical solution of an identification problem for observing two-phase flow in capillaries. Math. Methods Appl. Sci. 37, 2393-2405 (2014)

20. Erdogan, AS, Uygun, H: A note on the inverse problem for a fractional parabolic equation. Abstr. Appl. Anal. 2012, Article ID 276080 (2012)

21. Fatullayev, AF: Numerical procedure for the simultaneous determination of unknown coefficients in a parabolic equation. Appl. Math. Comput. 164, 697-705 (2005)

22. Isakov, V: Inverse Problems for Partial Differential Equations. Springer, New York (1998)

23. Ozbilge, E: Identification of the unknown diffusion coefficient in a quasi-linear parabolic equation by semigroup approach with mixed boundary conditions. Math. Methods Appl. Sci. 31, 1333-1344 (2008)

24. Ozbilge, E, Demir, A: Analysis of a semigroup approach in the inverse problem of identifying an unknown parameters. Appl. Math. Comput. 218, 965-969 (2011)

25. Ozbilge, E, Demir, A: Identification of the unknown coefficient in a quasi-linear parabolic equation by semigroup approach. J. Inequal. Appl. 2013, Article ID 212 (2013). doi:10.1186/1029-242X-2013-212

26. Ozbilge, E: Determination of the unknown boundary condition of the inverse parabolic problems via semigroup method. Bound. Value Probl. 2013, Article ID 2 (2013). doi:10.1186/1687-2770-2013-2

27. Ozbilge, E, Demir, A: Semigroup approach for identification of the unknown diffusion coefficient in a linear parabolic equation with mixed output data. Bound. Value Probl. 2013, Article ID 43 (2013). doi:10.1186/1687-2770-2013-43

\section{Submit your manuscript to a SpringerOpen ${ }^{\ominus}$ journal and benefit from:}

- Convenient online submission

- Rigorous peer review

- Immediate publication on acceptance

- Open access: articles freely available online

- High visibility within the field

- Retaining the copyright to your article 